\title{
Serum phosphorus levels in pulmonary tuberculosis
}

\author{
T. KARDJITO \\ M.D., Ph.D.
}

S. P. EDIYANTO

M.D.

\author{
J. M. GRANGE \\ M.Sc., M.D.
}

Faculty of Medicine, Airlangga University, Surabaya, Indonesia and the Cardiothoracic Institute, (University of London) Fulham Road, London SW3 6HP

\begin{abstract}
Summary
The mean level of phosphorus was significantly higher in a group of 96 Indonesian patients with pulmonary tuberculosis than in 100 control subjects. The levels in disease showed a moderate correlation with those of the globulin proteins, but not the $\gamma$-globulins. There was an association between levels of phosphorus and those of transferrin but not with those of seven other acute-phase associated proteins.

There was a tendency for phosphorus levels to be higher in those with elevated polymorphonuclear leucocyte counts and, although not significantly so, in those with more extensive active disease, suggesting an association with tissue destruction. It appears therefore more relevant to study the behaviour of this element than that of calcium in tuberculosis.
\end{abstract}

KEY WORDS: serum proteins, polymorphonuclear leucocyte.

\section{Introduction}

Before the advent of effective chemotherapy, numerous studies on the biochemistry of tuberculosis were carried out in the hope of finding some metabolic anomaly or defect whose rectification would lead to a cure. Thus calcium metabolism was investigated in the belief that calcification played an important role in the healing of lesions (Kaminsky and Davidson, 1931) but phosphorus has received relatively little attention. Wells, DeWitt and Long (1923) referred to a few early reports of phosphorus retention and reduced excretion and also to a few reports of increased urinary levels of lipid-bound phosphorus in patients with tuberculosis. Sweany, Weathers and McCluskey (1923-4) found that phosphorus levels varied considerably from patient to

Address for correspondence: Dr. J.M. Grange, Department of Microbiology, Cardiothoracic Institute, Fulham Road, London SW3 6HP, England. patient and they suggested that some of the phosphorus might be associated with lipoproteins liberated by the destruction of cell membranes. Recently Sharma $\omega$ (1981) observed elevated phosphorus levels in Indian patients who were hypercalcaemic but not in those 으 who were normocalcaemic.

In this study the levels of phosphorus in sera from $\subsetneq$ 96 Indonesian patients and 100 control subjects were determined in order to investigate further the behaviour of this element in tuberculosis.

\section{Materials and methods}

Sera were obtained from 96 Indonesian patients, aged $18-50$ years, with untreated pulmonary tubercu- $\frac{\circ}{\varnothing}$ losis and from 100 healthy and radiologically normal $\cong$ control subjects in the same age range and from the $\overrightarrow{0}$ same region. The blood samples were obtained in the morning after overnight fasting. Sera were separated from the blood clots within two hours of venepuncture and were stored at $-20^{\circ} \mathrm{C}$ prior to assay.

Serum phosphorus was assayed by use of a commercial test kit (Merckotest no. 3331, Merck), ํㅡㄹ calcium by the method of Clark and Collip (1925) and serum proteins (albumin, globulin and $\gamma$-globulin) according to Huerga, Smethers and Sherrick

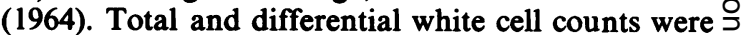
performed. The levels of total immunoglobulins in $\frac{D}{2}$ the IgG, IgM and IgA classes, antimycobacterial antibodies in these three classes and eight acute- $N$ phase reactant proteins $\left(\alpha_{1}\right.$-antitrypsin, $\alpha_{1}$-acid glycoprotein, $\alpha_{2}$-macroglobulin, C-reactive protein, caeru- $\bigcirc$ loplasmin, haptoglobin, transferrin and the third $\omega$ component of complement) were available from a previous study (Grange, Kardjito and Setiabudi, 응 1984).

The significance of differences between levels of the various serum components were determined by Student's $\boldsymbol{t}$ test and Mann Whitney test and correla- 
TABLE 1. Means and standard deviations (s.d.) of the levels of phosphorus, calcium and the serum proteins in the patients and control subjects

\begin{tabular}{lcccccc}
\hline & \multicolumn{2}{c}{ Patients } & & \multicolumn{2}{c}{ Controls } & \\
\cline { 2 - 3 } & Mean & s.d. & & Mean & s.d. & Significance of difference* \\
\hline Phosphorus (mmol/l) & 1.51 & 0.48 & & 1.31 & 0.29 & $<0.001$ \\
Calcium (mmol/l) & 2.37 & 0.16 & & 2.36 & 0.17 & NS \\
Albumin (g/dl) & 4.91 & 0.70 & & 4.88 & 0.58 & NS \\
Globulin (g/dl) & 2.88 & 0.69 & & 2.40 & 0.50 & $<0.001$ \\
$\gamma$-globulin (g/dl) & 0.80 & 0.34 & & 0.60 & 0.18 & $<0.001$ \\
\hline
\end{tabular}

*NS $=$ not significant $(P>0.05)$.

tion coefficients were calculated by Spearman's method in which $1=$ perfect correlation, $-1=$ perfect inverse correlation and $0=$ no correlation.

The chest radiographs were read by Professor J. G. Scadding and the extent of active disease was staged as minimal (less than 2 lung zones), moderate (2-4 lung zones) and extensive (more than 4 lung zones). The phosphorus levels in these three groups were compared by one-way variance analysis.

\section{Results}

Mean levels and standard deviations of serum phosphorus, calcium, and the proteins are shown in Table 1. There were no significant differences in the levels of calcium and albumin between the patient and control groups while those of phosphorus, globulin and $\gamma$-globulin were significantly higher in the former group. The range of calcium levels was very similar in patients and controls (2.05-2.69 and 2.02-2.70 mmol/1 respectively). The range of phosphorus levels was greater in patients $(0 \cdot 76-3 \cdot 23$ $\mathrm{mmol} / \mathrm{l})$ than in controls $(0.88-2.04 \mathrm{mmol} / \mathrm{l})$. Twelve patients had levels above the mean + two standard deviations of the control levels.

There was no correlation between phosphorus levels and those of the proteins or calcium in the control group but in the patient group there was a low but significant inverse correlation between phosphorus and albumin levels $(r=-0 \cdot 20, P<0 \cdot 01)$ and a moderate direct correlation of phosphorus to globulin levels $(r=0.47, P<0.001)$ but not to $\gamma$-globulin levels $(r=0.05)$. In both patients and controls there was an inverse correlation between albumin and globulin levels $(r=0.50$ and 0.60 respectively, $P<0.001)$. The inverse correlation between albumin and phosphorus levels among the patients was secondary to that between albumin and globulin; when the latter effect was removed the former correlation was lost (partial $r=0.06$ ).

Phosphorus levels did not correlate significantly with those of the total immunoglobulins or antimycobacterial antibodies in the IgG or IgM classes but there was a low inverse correlation with total IgA among the patients $(r=-0.23, P<0.05)$ and the controls $(r=-0 \cdot 20, P<0.05)$. Levels of phosphorus were related to those of transferrin in the patients $(r=0.32, P<0.01)$ but not in the controls $(r=0.09)$. The other acute phase reactants showed no signficant correlations with the phosphorus levels.

There was a low but significant correlation between the polymorphonuclear leucocyte count and phosphorus levels among the patients $(r=0.23$, $P<0.05)$ but not among the controls $(r=0.08)$.

The extent of radiologically assessed active disease was minimal in 23 patients, moderate in 44 patients and extensive in 28 patients. The mean (and standard deviations) of the phosphorus levels in these groups were $1.41(0.37), 1.49(0.35)$ and $1.60(0.70) \mathrm{mmol} / 1$ respectively. Although the levels increased with the extent of disease this, on variance analysis, was not statistically significant.

\section{Discussion}

Although more attention has previously focussed on calcium than on phosphorus in tuberculosis, this study suggests that the latter is much more affected than the former in this disease. The levels of phosphorus were associated with those of globulins, but not those of the $\gamma$-globulins, in the patients only but the reason for this association is unknown. It may, however, indicate the increased synthesis, in tuberculosis, of a protein in the $\alpha$ - or $\beta$-globulin classes that affects the metabolism or excretion of phosphorus. The levels of this element did not, however, correlate with those of eight acute-phase and related proteins (with the exception of transferrin) and it is thus possible that it is associated with certain lipoproteins liberated as a result of tissue destruction as suggested by Sweany et al. (1923-4). This concept may explain the low but significant correlation of phosphorus levels and the polymorph count among patients and also the tendency, albeit not a statistically significant one, for phosphorus levels to be related to the extent of active disease as determined radiologically.

There was no difference in calcium levels in the patients and controls and no significant correlation 
between the levels of this element and those of phosphorus. Thus, in contrast to the findings of Sharma (1981), elevated phosphorus levels were not restricted to those with hypercalcaemia.

In conclusion, phosphorus levels tend to be elevated in Indonesian patients with tuberculosis but whether this finding is common to patients throughout the world and whether it has a primary bearing on the immunopathology of the disease remains to be determined.

\section{Acknowledgments}

We thank Professor J. G. Scadding for reading the chest radiographs and Drs B. Cheng and P. D. O. Davies for their critical comments. We are also grateful to the British Council for financial assistance to one of us (T.K.).

\section{References}

ClarK, E.P. \& ColliP, J.B. (1925) A study of the Tisdall method for the determination of blood serum calcium with a suggested modification. Journal of Biological Chemistry, 63, 461.

Huerga, J. De LA, Smethers, G.W. \& SherRick, J.C. (1964) Colorimetric determinations of serum proteins. In: Serum Proteins and Dysproteinaemias. (Eds. F. W. Sunderman and F. W. Sunderman, Jr.) Lippincott Co., Philadelphia.

Grange, J.M., Kardjito, T. \& Setiabudi, I. (1984) A study of acute phase reactant proteins in Indonesian patients with pulmonary tuberculosis. Tubercle 65, 23.

KARMinSKY, J. \& DAvidSON, D.L. (1931) Serum calcium in pulmonary tuberculosis. American Review of Tuberculosis, 23, 422.

SHARMA, S.C. (1981) Serum calcium in pulmonary tuberculosis. Postgraduate Medical Journal, 57, 694.

SWEANY, H.C., Weathers, A.T. \& McCluskey, K.L. (1923-4) ڤે The chemistry of blood in tuberculosis. American Review of $\vec{O}$ Tuberculosis, 8, 405 .

WELLS, H.G., DEWITT, L.M. \& LONG, E.R. (1923) The chemistry of tuberculosis. Bailliere, Tindall and Cox, London.

(Accepted 10 November 1983) 